\title{
Bilateral renal angiomyolipomas in tuberous sclerosis
}

\author{
Bushra Jawaid*, Abdul Hafeez Qureshi, Nadeem Ahmed and Nousheen Yaqoob
}

\begin{abstract}
Background: Tuberous sclerosis complex (TSC) is a rare genetic disorder characterized by benign hamartomas in multiple organs of the body. Renal angiomyolipomas (AML) are commonly associated with TSC. They are mostly asymptomatic. But large and rapidly growing AMLs with the presence of an aneurysm cause symptoms and pose a life-threatening risk for hemorrhage.

Case presentation: Our patient is a 25-year-old female who presented to us as an undiagnosed case of tuberous sclerosis having a large abdominal mass. She fulfilled the clinical criteria required for the diagnosis of TSC. The CT scan revealed an $18 \times 13 \times 33 \mathrm{~cm}$ fat-containing lesion in the right kidney with an adjacent aneurysm measuring around $16 \mathrm{~cm}$ in diameter. Due to the large size of the AML and associated aneurysm, surgical exploration was mandated. On the contralateral kidney, multiple contrast-enhancing soft-tissue densities were present that appeared suspicious on radiology. So a percutaneous biopsy of those lesions was done. Fortunately, it had the same histopathology as an Angiomyolipoma. Nephrectomy of the right-sided kidney with AML has been done. The left-sided lesions that are less than $2 \mathrm{~cm}$ and asymptomatic are kept on close surveillance. Any change in size will prompt therapy with mTOR inhibitors.
\end{abstract}

Conclusion: When dealing with bilateral renal AML, it is important to adopt a conservative approach. When intervention is indicated, the least invasive strategy should be sought and enacted. Radical surgery should be the last resort.

Keywords: Angiomyolipoma, Tuberous sclerosis, Bilateral renal angiomyolipoma, MTOR inhibitors

\section{Background}

Tuberous sclerosis (TS), also known as BournevillePringle disease, is a rare genetic disease with an autosomal dominant pattern of inheritance [1]. The disease manifest as benign tumors in various organs like the retina, lungs, heart, brain, skin, and kidney. The kidney is affected in approximately $80 \%$ of these patients with $75 \%$ of the lesions being angiomyolipomas. Despite having a benign nature, these tumors are the most common cause of TSC-related death because of spontaneous hemorrhage leading to hypovolemic shock and renal failure requiring long-term dialysis or transplant. We report

${ }^{*}$ Correspondence: BushraJ.Kurd@gmail.com

The Indus Hospital Karachi, Korangi Crossing, Karachi 75190, Pakistan a case of bilateral renal angiomyolipomas largest being $18 \mathrm{~cm}$ with a bleeding aneurysm.

\section{Case presentation}

A 25-year-old female, married, with no prior comorbidities, presented with the complaint of an abdominal mass for the past eight months. Initially, it was noticed as a small bulge in the right flank and was associated with occasional discomfort. It progressively increased in its size, involving the entire right-sided abdomen with extension across the midline, associated with episodes of moderate-intensity pain requiring oral analgesics.

The patient had multiple small lesions on her face since adolescence. She denied any significant medical or surgical history in the past. There was not any history of altered bowel habits or any other GI-related symptoms. She did not have any urinary complaints or 


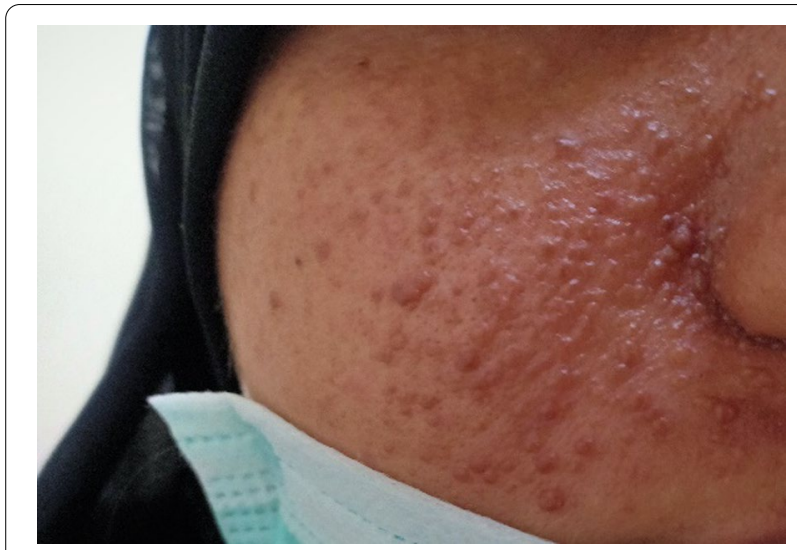

Fig. 1 Fascial angiofibromas

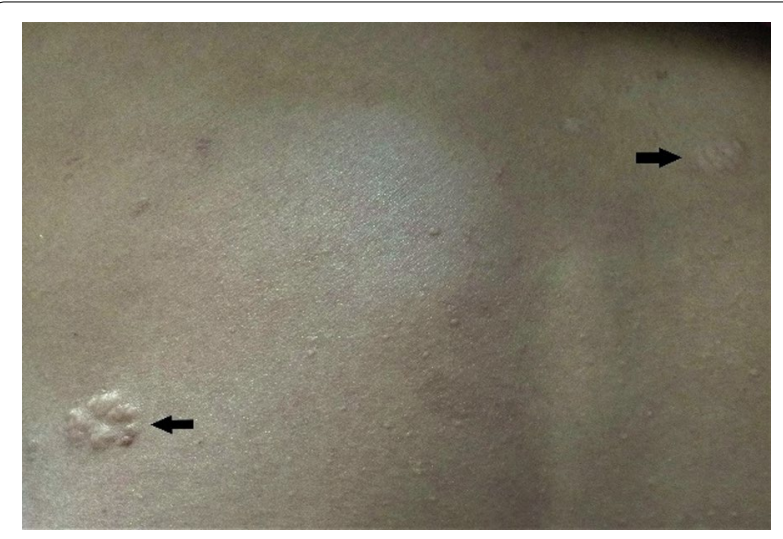

Fig. 2 'Orange peel' appearance of a shagreen patch

a history of hematuria. She did not have any history of seizures or developmental delays. She denied any history of chest pains, palpitations, or syncopal attacks. Did not have any chronic cough or history of wheezing. There isn't any known genetic disease in her family.

On general physical examination, she was a young female with average height and build and a grossly visible abdominal protuberance. Vital signs were within the satisfactory limit. She was clinically pale with multiple well-defined, brown sessile nodular growths on her nose and cheeks in a characteristic "butterfly pattern" (Fig. 1). She had a well-defined hypopigmented patch on the lower back showing an orange peel appearance indicative of a shagreen patch (Fig. 2). On abdominal examination, she had a large globular mass extending from the right flank crossing the midline up to the left lumbar region with a lower limit palpable up to the iliac fossas.

It was firm, with a smooth surface, ballotable, non-tender, and non-pulsatile.

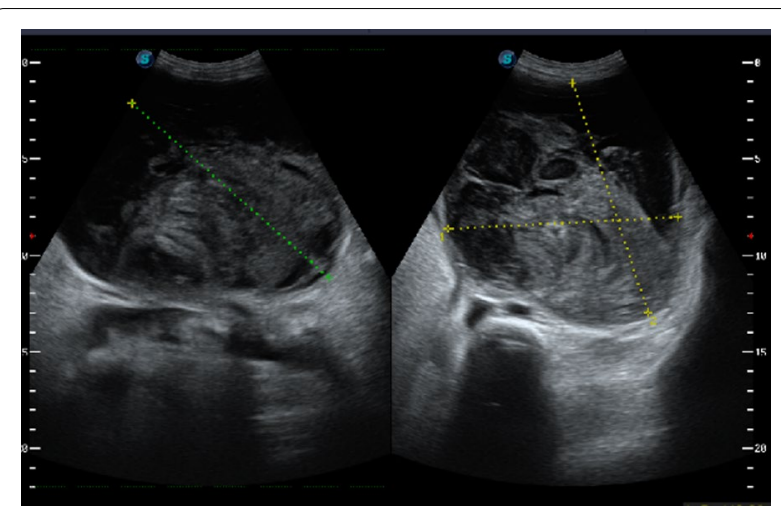

Fig. 3 Ultrasound images of a heterogeneous mass lesion measuring $11.6 \times 12.9 \times 13.6 \mathrm{~cm}$ in mid abdomen extending into lower abdomen

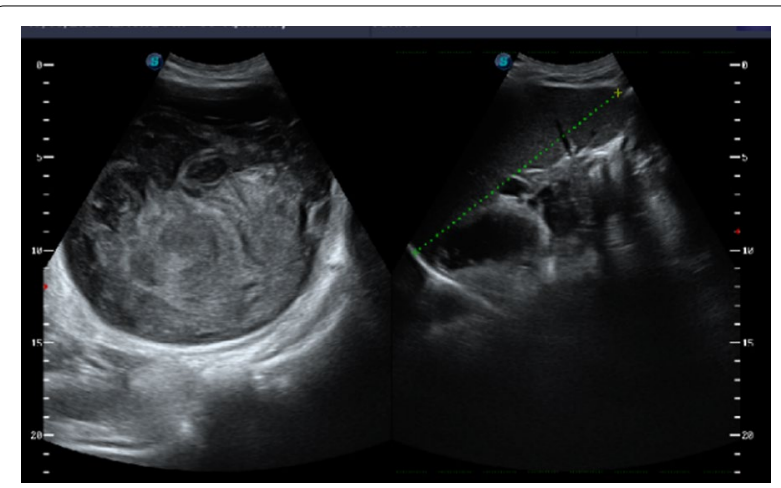

Fig. 4 Ultrasound images of a heterogeneous mass lesion measuring $11.6 \times 12.9 \times 13.6 \mathrm{~cm}$ in mid abdomen extending into lower abdomen

On initial laboratory workup, she was found to be severely anemic with a hemoglobin of $5.1 \mathrm{mg} / \mathrm{dl}$. Ultrasound showed a large heterogeneous mass lesion measuring $11.6 \times 12.9 \times 13.6 \mathrm{~cm}$ in mid-abdomen extending into the lower abdomen. The right kidney could not be visualized (Figs. 3, 4).

A contrast-enhanced CT scan of abdomen and pelvis was done for further characterization of the renal masses. A huge heterogeneous fat-containing lesion was seen arising from the interpolar region of the right kidney with an adjacent large hypodense lesion with internal high density and a well-defined contrast filling on its medial aspect, closely abutting the right renal lesion. These findings were suggestive of a huge right-sided renal angiomyolipoma with a bleeding pseudoaneurysm. Few enhancing soft tissue densities were found in the angiomyolipoma of the left kidney larges $2.2 \times 2.3 \mathrm{~cm}$ raising the suspicion of renal cell carcinoma (Figs. 5, 6).

According to the 2012 International tuberous sclerosis complex consensus conference, TCS can be diagnosed 


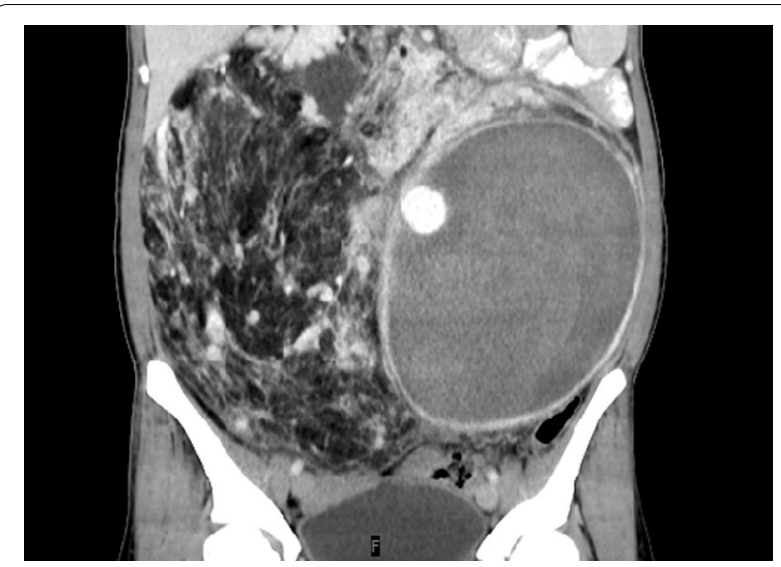

Fig. 5 Huge right-sided renal angiomyolipoma with a bleeding pseudoanurysm. Few hyperdense enhancing soft tissue densities were noted in the angiomyolipoma of the left kidney

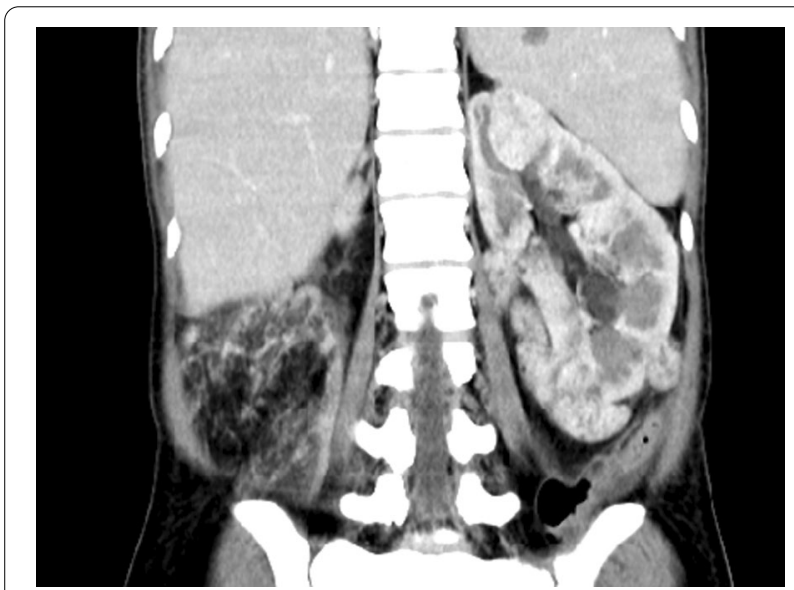

Fig. 6 Huge right-sided renal angiomyolipoma with a bleeding pseudoanurysm. Few hyperdense enhancing soft tissue densities were noted in the angiomyolipoma of the left kidney

based on "genetic diagnostic criteria" or "clinical diagnostic criteria" [2]. The genetic diagnosis requires the identification of a TSC1 or a TSC2 pathogenic mutation in DNA from normal tissue. The clinical diagnostic criteria include 11 major and six minor features. For a definite diagnosis two major features or one major feature with $>2$ minor features are required. Our patient had four major features, i.e., hypomelanotic macules, angiofibromas, shagreen patch, and angiomyolipomas. Hence, the definitive diagnosis of TCS was made.

The multiple enhancing lesions of the left kidney, although $<4 \mathrm{~cm}$, had confusing features on $\mathrm{CT}$ and mandated a biopsy. An ultrasound-guided biopsy of the left renal lesion was done. Fortunately, the histopathology confirmed AML. A nephrectomy was planned for

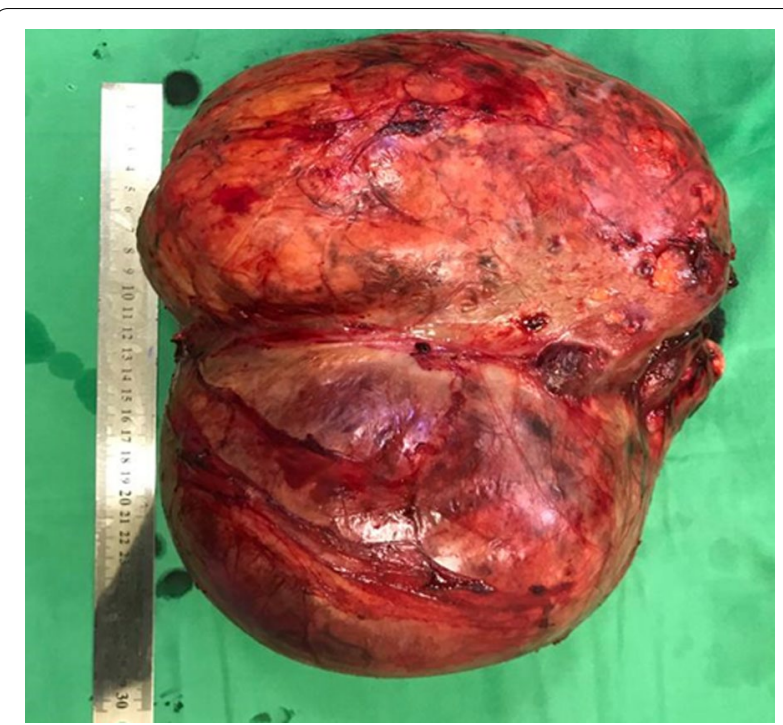

Fig. 7 Right-sided kidney with AML measuring $26 \times 23 \times 15 \mathrm{~cm}$. Renal tissue could not be separately visualized

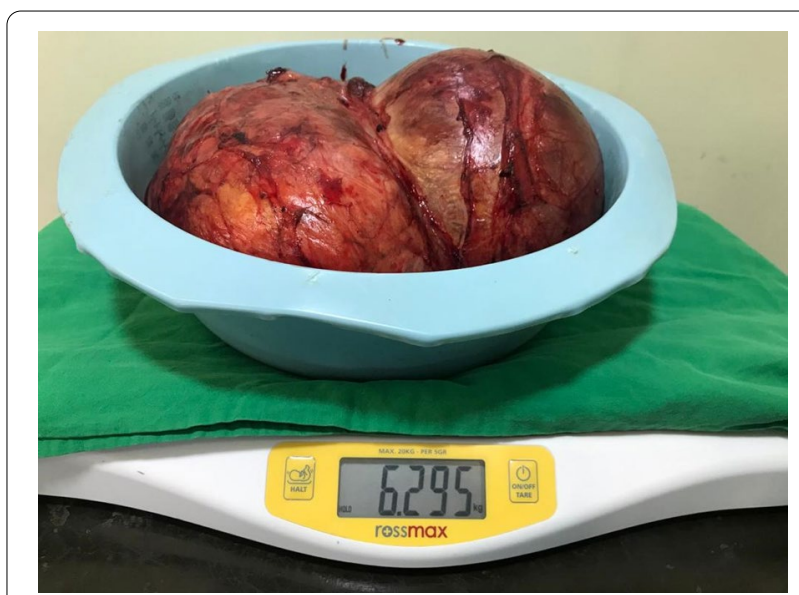

Fig. 8 Right-sided kidney with AML measuring $26 \times 23 \times 15 \mathrm{~cm}$. Renal tissue could not be separately visualized

the right-sided renal mass. A midline laparotomy incision was given, extending from xiphisternum to pubic symphysis.

A Huge mass, measuring $26 \times 23 \times 15 \mathrm{~cm}$ involving the middle and lower pole of the right kidney with a large cystic component extending across the midline and causing the medial shift of ascending colon, was found. Its large size and more than two-thirds involvement of the kidney mandated complete nephrectomy. The tumor was highly vascular with multiple dilated peri-tumoral vessels requiring meticulous mobilization and ligation (Figs. 7, 8). 


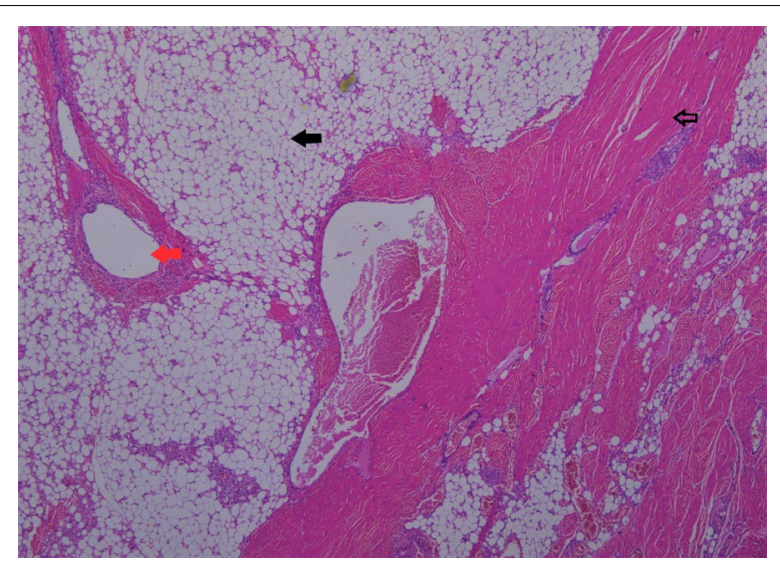

Fig. 9 Thick-walled blood vessels (solid red arrow) surrounding smooth muscle fibers (empty black arrow) and interposed adipocytes (solid black arrow)

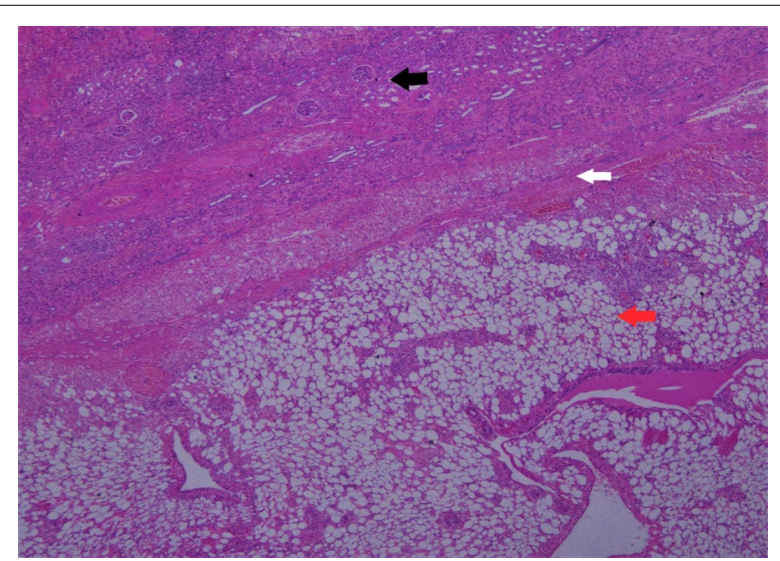

Fig. 10 AML (red arrow), normal renal parenchyma with glomerulus (black arrow), well circumscribed margin between AML and normal renal tissue (white arrow)

The specimen was sent for histopathological examination. Microscopic examination revealed multiple thick-walled blood vessels surrounded by radial arrays of smooth muscle fibers and admixed among mature adipocytes (Fig. 9).

The neoplastic cells were positive for HMB45, MelanA, and Alpha smooth muscle actin immunohistochemical markers. The tumor was well-circumscribed with no infiltration in the adjacent renal parenchyma (Fig. 10). Features were consistent with renal angiomyolipoma.

We are following our patient with an ultrasound every six months. So far, she is asymptomatic, and no increase in the size of the lesions in her left solitary kidney is noted.

\section{Discussion}

Tuberous sclerosis (TS) is a rare genetic disease with an autosomal dominant pattern of inheritance and arises from the inactivation of either TSC1 (chromosome locus 9q34.3) or TSC2 (16p13.3) genes [2]. These genes are responsible for the formation of a protein "hamartin" (TSC1) and "tuberin" (TSC2), which together form a tumor suppressor complex and limit abnormal cell proliferation. The disease manifest as benign tumors in various organs, including the retina, lungs, heart, brain, skin, and kidney [3]. Neuropsychiatric disorders are also associated with TSC $[4,5]$. Patients having TSC can develop different epithelial renal lesions, including polycystic kidney disease (PKD), oncocytomas, and renal cell carcinomas (RCC), but angiomyolipomas remain the most common. These are mostly asymptomatic and discovered incidentally, but lesions more than $4 \mathrm{~cm}$ may cause symptoms (e.g., hemorrhage, palpable mass, or symptoms related to mass effect) hence requiring treatment $[6,7]$.

CT scan remains the most accurate modality for the characterization and diagnosis of angiomyolipoma. They are classified as "classical" and "atypical" based on their fat component. Although RAML has a characteristic feature of fat attenuation on CT scan, the presence of intratumoral calcifications, intratumoral cysts, or a solitary fat-poor AML increases the suspicion of malignancy and requires a biopsy $[8,9]$.

Treatments preserving renal function should be considered for every patient with AML given its benign nature. Symptomatic lesions or tumors greater than $4 \mathrm{~cm}$ in diameter are treated with surgical resection or selective renal artery embolization [7].

In the last few decades, there has been a tremendous amount of work done in recognizing the role of mTOR inhibitors in the management of renal AML. Inhibitors of the mTOR pathway such as rapamycin, also known as sirolimus, with their immunosuppressive and antiproliferative actions, have shown a therapeutic benefit. The mutation of the TSC genes leads to the unregulated activity of mTORC1 with subsequent unabated growth of hamartomas. These mTOR inhibitors can block this pathway directly at the level of mTORC1 resulting in up to $50 \%$ reduced angiomyolipoma volume with an acceptable safety profile $[10,11]$.

Renal AML associated with TCS grows at an estimated rate of $1.25 \mathrm{~cm}$ yearly. Therefore, a lifetime follow-up is needed [12]. To date, there are no strict criteria of how frequently these should be imaged and so the current practice is an individualized surveillance protocol based on the clinical background, i.e., the size of the lesion and whether the patient has TSC. 


\section{Conclusion}

Our experience concludes that bilateral angiomyolipomas with such an atypical presentation should be investigated thoroughly and whenever in doubt, a biopsy should be done before making any management decisions. The aim should be at preserving renal function with a least invasive approach.

\section{Abbreviations}

TSC: Tuberous sclerosis complex; AML: Angiomyolipomas; TS: Tuberous sclerosis; PKD: Polycystic kidney disease; RCC: Renal cell carcinomas.

\section{Acknowledgements}

We thank our patient for allowing us to share her details, the radiologist for providing valuable help in diagnosing the condition and the co-authors for their expertise and assistance throughout the process.

\section{Authors' contributions}

BJ has done the research work related to the topic and has done the manuscript writing. AH and NA made the clinical decision of the case and have helped to draft the manuscript. NY is the histopathologist who reviewed the biopsy specimens from this case. She provided valuable help and guidance to us for a timely diagnosis. All authors have read and approved the manuscript and have given approval of the version to be published.

\section{Funding}

There is no funding to declare.

\section{Availability of data and material}

The data mentioned in the case report study is available from the corresponding author upon reasonable request.

\section{Declarations}

Ethics approval and consent to participate

An informed written consent was taken from the subject of discussion prior to submission.

\section{Consent for publication}

An informed written consent was taken from the patient for publication of case.

\section{Competing interests}

The authors declare that there are no conflict of interest.

Received: 16 December 2020 Accepted: 29 March 2021

Published online: 13 April 2021

\section{References}

1. Shankar S, Hulikanthimatt KS, Awatti SM, Narayanrao ST (2015) Bilateral multifocal renal angiomyolipoma associated with wunderlich's syndrome in a tuberous sclerosis patient. J Clin Diagn Res. 9(8):ED01-3. https://doi. org/10.7860/JCDR/2015/13349.6289

2. Northrup $H$, Krueger DA, International Tuberous Sclerosis Complex Consen (2013) Tuberous sclerosis complex diagnostic criteria update: recommendations of the 2012 international tuberous sclerosis complex consensus conference. Pediatr Neurol. 49(4):243-254. https://doi.org/10. 1016/j.pediatrneurol.2013.08.001

3. Caban C, Khan N, Hasbani DM, Crino PB (2016) Genetics of tuberous sclerosis complex: implications for clinical practice. Appl Clin Genet 21(10):1-8. https://doi.org/10.2147/TACG.S90262

4. Zöllner JP, Franz DN, Hertzberg C et al (2020) A systematic review on the burden of illness in individuals with tuberous sclerosis complex (TSC). Orphanet J Rare Dis 15:23. https://doi.org/10.1186/s13023-019-1258-3

5. Jeong A, Wong M (2016) Systemic disease manifestations associated with epilepsy in tuberous sclerosis complex. Epilepsia 57:1443-1449. https:// doi.org/10.1111/epi.13467

6. Zimmerhackl LB, Rehm M, Kaufmehl K, Kurlemann G, Brandis M (1994) Renal involvement in tuberous sclerosis complex: a retrospective survey. Pediatr Nephrol 8(4):451-457. https://doi.org/10.1007/BF00856529

7. Steiner MS, Goldman SM, Fishman EK, Marshall FF (1993) The natural history of renal angiomyolipoma. J Urol 150(6):1782-1786. https://doi.org/ 10.1016/s0022-5347(17)35895-0

8. Park BK (2017) Renal angiomyolipoma: radiologic classification and imaging features according to the amount of fat. AJR Am J Roentgenol 209(4):826-835. https://doi.org/10.2214/AJR.17.17973

9. Halpenny D, Snow A, McNeill G, Torreggiani WC (2010) The radiological diagnosis and treatment of renal angiomyolipoma-current status. Clin Radiol 65(2):99-108. https://doi.org/10.1016/j.crad.2009.09.014

10. Curatolo P, Bjørnvold M, Dill PE, Ferreira JC, Feucht M, Hertzberg C, Jansen A, Jóźwiak S, Kingswood JC, Kotulska K, Macaya A, Moavero R, Nabbout R, Zonnenberg BA (2016) The role of mTOR inhibitors in the treatment of patients with tuberous sclerosis complex: evidence-based and expert opinions. Drugs 76(5):551-565. https://doi.org/10.1007/ s40265-016-0552-9

11. Trnka P, Kennedy SE (2020) Renal tumors in tuberous sclerosis complex. Pediatr Nephrol. https://doi.org/10.1007/s00467-020-04775-1

12. Seyam RM, Bissada NK, Kattan SA, Mokhtar AA, Aslam M, Fahmy WE, Mourad WA, Binmahfouz AA, Alzahrani HM, Hanash KA (2008) Changing trends in presentation, diagnosis and management of renal angiomyolipoma: comparison of sporadic and tuberous sclerosis complex-associated forms. Urology 72(5):1077-1082. https://doi.org/10.1016/j.urology. 2008.07.049

\section{Publisher's Note}

Springer Nature remains neutral with regard to jurisdictional claims in published maps and institutional affiliations.

\section{Submit your manuscript to a SpringerOpen ${ }^{\circ}$ journal and benefit from:}

- Convenient online submission

$\checkmark$ Rigorous peer review

- Open access: articles freely available online

- High visibility within the field

- Retaining the copyright to your article

Submit your next manuscript at $\boldsymbol{\nabla}$ springeropen.com 